\title{
Foreword: Structural Transitions and Local Deformation Processes at and Near Grain Boundaries
}

This volume contains a collection of articles reporting research that was presented at the recent symposium "Structural Transitions and Local Deformation Processes at and Near Grain Boundaries" held at the MS\&T meeting in Fall 2009 in Pittsburgh, PA. The reader is also referred to the December 2009 issue of $J O M$ (vol. 61, pp. 37-58) in which three articles describe additional research that was presented at this symposium.

Several aspects of grain boundary influence are explored in this issue. Grain boundary character continues to be an important strategy for optimizing the performance of structural and functional materials. The distribution of grain boundary character evolves with thermal activation that minimizes system energy. Grain boundary diffusion has a large impact on high-temperature deformation and second-phase evolution. Temperature and composition (minority elements) can dramatically affect grain boundary mobility or the ability to slide under the influence of stress. Nanoscale structural features in both natural and man-made structures are examined for their influence on material properties. Heterogeneous deformation at the microstructural scale of polycrystals originating from grains or phases that have different elastic and plastic properties with respect to applied stress deform differently leading to strain gradients and substructure evolution within grains. Thus, the structure of the interfaces strongly influences deformation behavior that causes variations in properties. Mechanical twinning is also an important component in microstructural evolution, generating new low-energy interfaces with transformation and deformation that affect strength and ductility.

Many of these articles illustrate new experimental and analytical methods to quantify defect content at or near grain boundaries, especially in combined or synergistic approaches to characterize and model heterogeneous deformation. Atomistic, continuum, and crystal plasticity approaches to modeling are examined in the context of grain boundaries to realistically incorporate the properties of grain boundaries into simulative models. In some cases, simulations are directly compared with highly characterized regions of microstructure. New or novel uses of characterization techniques include surface profilometry, electron backscatter pattern mapping, transmission electron microscopy, and three-dimensional X-ray microdiffraction microscopy, which allows for identification of local crystal orientation, orientation gradients, defect density, and internal strains at the micron scale. These tools allow investigation of a subtle microstructural evolution mechanism that governs grain boundary mobility, recrystallization, and damage nucleation; thus, the foundation is laid for developing rules that will give guidance on modifying processing paths to improve material performance and reliability prediction.

We hope that you will find these articles interesting and inspirational, and perhaps they will lead to new results and insights that can be shared at the follow-up symposium titled "Deformation and Transitions at Grain Boundaries" at the MS\&T meeting October 16-21, 2011 in Columbus, OH. Watch for invitations for abstracts that will be due in March 2011.

Symposium Organizers:

Thomas R. Bieler

Chemical Engineering and Materials Science

Michigan State University East Lansing, MI 48824-1226

\author{
Rozaliya Barabash
}

University of Tennessee, Oak Ridge National Laboratory P.O. Box 2008 Oak Ridge, TN 37831-6118

Stephen W. Banovic

Gaithersburg Safety, Health, and Environment Division

National Institute of Standards and Technology, Office of Safety, Health, and Environment Bldg. 301, Room B120, 100 Bureau Drive, MS 1730, Gaithersburg, MD 20899 